\title{
DISTROFIA POLIMORFA POSTERIOR DE LA CÓRNEA ASOCIADA CON DISTROFIA RETINIANA DE CONOS
}

Juan F. Diaz Granados MD*, Andrés Reyes MD MSc**, José F. Arango MD***

\section{Resumen}

La medicina actual enfoca las enfermedades hacia el nivel molecular y genético, y toda la comunidad científica debe colaborar con tal fin. Cuando encontramos patologías de origen genético con variaciones no publicadas antes, es nuestro deber informarlo y así ayudar a su conocimiento. Reportamos un paciente de 43 años con historia de disminución progresiva de la visión por ambos ojos desde la niñez. Sus antecedentes familiares son negativos. El examen oftalmológico reveló mala agudeza bilateral, lesiones corneanas compatibles con DPP de la córnea y en la fundoscopia una imagen en "ojo de buey".

Se solicitaron estudios complementarios de campimetría macular, electrorretinograma, angiografía fluoresceínica y prueba de colores Farnsworth Munsell 15D, pruebas que demostraron alteraciones en los conos de la mácula corroborando el diagnóstico de DRC.

La prevalencia de la DPPy de la DRC es muy baja y no hemos encontrado hasta la fecha ningún reporte de la literatura en que se presenten en un solo paciente. Esto abre las puertas a nuevas inquietudes como ¿Existe relación genética entre las dos alteraciones? Si los antecedentes familiares son negativos, ¿Las dos patologías son fruto de una mutación de novo? Esperamos en un futuro no muy lejano poder responder estas preguntas.

Palabras clave: distrofia de córnea, distrofia retiniana de conos, oftalmología.

Abreviaturas: DPP, distrofia polimorfa posterior; DRC; distrofia retiniana de conos.

\section{Justificación}

Con los avances tecnológicos en la medicina, cada día se estudia más a nivel molecular y genético las enfermedades, así en un futuro probablemente será posible prever muchas de éstas y en otros casos manipular genéticamente nuestro código para curarlas. Es así como hoy en día es importante observar, estudiar e investigar todo aquello que pueda poner al descubierto, hallazgos que ayuden nuestra comunidad científica para lograr tal fin. En este trabajo queremos reportar la presencia de dos patologías independientes, con etiologías genéticas que afectan tejidos diferentes del

Fecha recibido: agosto I de 2006 -Fecha aceptado: agosto 25 de 2006

* Profesor de córnea y segmento anterior, jefe del servicio de oftalmología Hospital de San José. Profesor Asistente Fundación Universitaria de Ciencias de la Salud.

** Instructor Asociado de cirugía vitreorretiniana, Fundación Universitaria de Ciencias de la Salud.

*** Residente I, oftalmología Fundación Universitaria de Ciencias de la Salud ojo y que según nuestro conocimiento, no han sido reportadas en la literatura mundial asociadas en un mismo individuo: distrofia polimorfa posterior de la córnea y distrofia retiniana de conos.

\section{Reporte de caso}

Un paciente de 43 años consulta por disminución progresiva de la visión en ambos ojos desde la niñez, la cual ha ido progresando, asociado a fotofobia. Niega antecedentes personales y familiares. En el examen oftalmológico se encuentra visión de cuenta dedos en ambos ojos que con refracción mejora a 20/400. Al examen de segmento anterior en lámpara hendidura se observan como dato positivo, unas lesiones lineales blanco-grisáceas localizadas en la membrana de Descemet en la córnea de ambos ojos (Figura 1). 

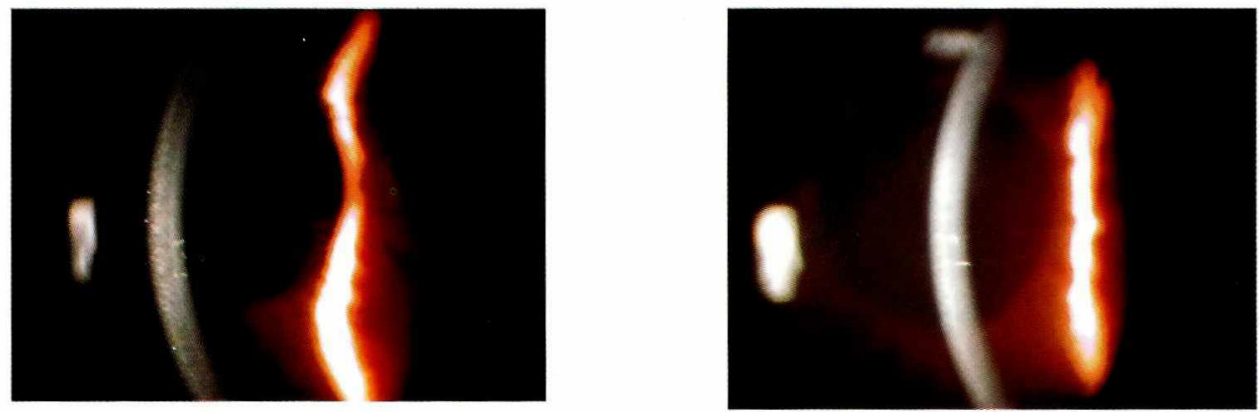

Figura I. Se evidencian bandas horizontales grisáceas en superficie posterior de ambas córneas, imágenes compatibles con DPP.

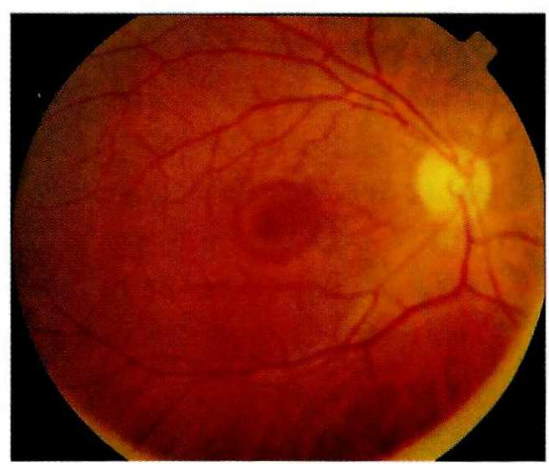

Ojo derecho

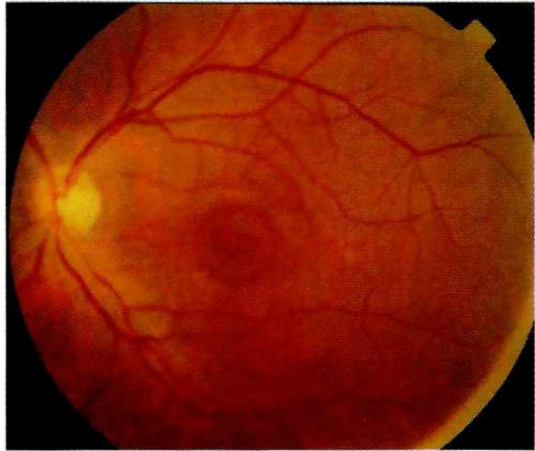

Ojo izquierdo

Figura 2. Fondo de ojo donde se evidencia una atrofia del epitelio pigmentario en forma anular a nivel macular en ambos ojos dando la apariencia de un "ojo de buey".

La presión intraocular fue de $12 \mathrm{mmHg}$. en ambos ojos. En el examen de fundoscopia se observó una atrofia anular del epitelio pigmentario de la retina macular, conocida como imagen en "ojo de buey" (Figura 2).

Con estos hallazgos se hace una impresión diagnóstica de distrofia de conos y distrofia polimorfa posterior, una asociación de patologías que no está reportada en la literatura.

Se solicitaron examenes complementarios, campimetría Humphrey 10-2 umbral completo, electroretinograma, angiografía fluoresceínica y prueba de colores Farnsworth Munsell 15D.

En la campimetría (Figura 3), se evidenció el compromiso macular presentando un aumento de la mancha ciega y un escotoma arqueado en el campo visual temporal de la mácula derecha y un escotoma superonasal en el izquierdo además de tener una disminución generalizada de la sensibilidad en toda la mácula de ambos ojos.
El electrorretinograma mostró una ausencia de las onda B y una amplitud disminuída en la onda A en la porción mesópica de ambos ojos. En las porciones fotopicas y flicker no se obtuvo respuesta registrable en ambos ojos. Lo anterior evidencia una alteración retiniana generalizada que afecta tanto las células de Müller como la función de conos centrales (foveales) de ambos ojos compatible con una distrofia bilateral (Figura 4).

En la angiografía fluoresceínica se observa un llenado coroideo de base regular con transito del medio sin retardo ni escapes en ambos ojos. En la zona foveal se observan efectos de pantalla por leve hiperplasia difusa del epitelio pigmentario de la retina (Figura 5) en la zona foveal se observa un area de hiperfluorescencia central rodeada de un area de no fluorescencia.

Los hallazgos de la angiografía fluoresceínica muestra una alteración del complejo epitelio pigmentario de la retina (EPR)-membrana de Bruch que es compatible con nuestro diagnóstico de distrofia retiniana de conos. 
Se realizó una prueba de Farnsworth Munsell 15D para valorar la discriminación al color la cual está alterada en la distrofia de conos. EN esta prueba se evidenció la gran alteración del paciente paa la discriminación de colores. No hubo un patrón definido en los ejes protan o deutan lo cual es compatible con la distrofia de conos (Figura 6).

\section{Díscusión}

La prevalencia de la distrofia polimorfa posterior y de la distrofia de conos es muy baja. Son patologías que en presentación independiente se consideran raras y hasta el momento no hemos encontrado ningún reporte en la literatura en el que se presenten las dos distrofias en un paciente. Con este caso pueden surgir inquietudes interesantes que nos motiven a indagar e investigar más sobre los aspectos etio-patogénicos y genéticos de estas dos entidades. También esperamos en un futuro no muy lejano responder preguntas como ¿Cual es el patrón genético de cada una de estas entidades en el paciente si sus antecedentes familiares son negativos? ¿Puede ser una presentación esporádica? ¿Una mutación de novo? ¿Hay alguna relación entre la alteración genética de la distrofia polimorfa posterior de la cornea y la distrofia retiniana de conos? El paciente además tiene una hija de dos años y se recomendó una evaluación oftalmológica, para así detectar si tiene alguna de estas patologías y demostrar si hay o no transmisión genética de una o de ambas patologías en su familia.

Es importante continuar estudiando el paciente de manera multidisciplinaría. En conjunto con el servicio

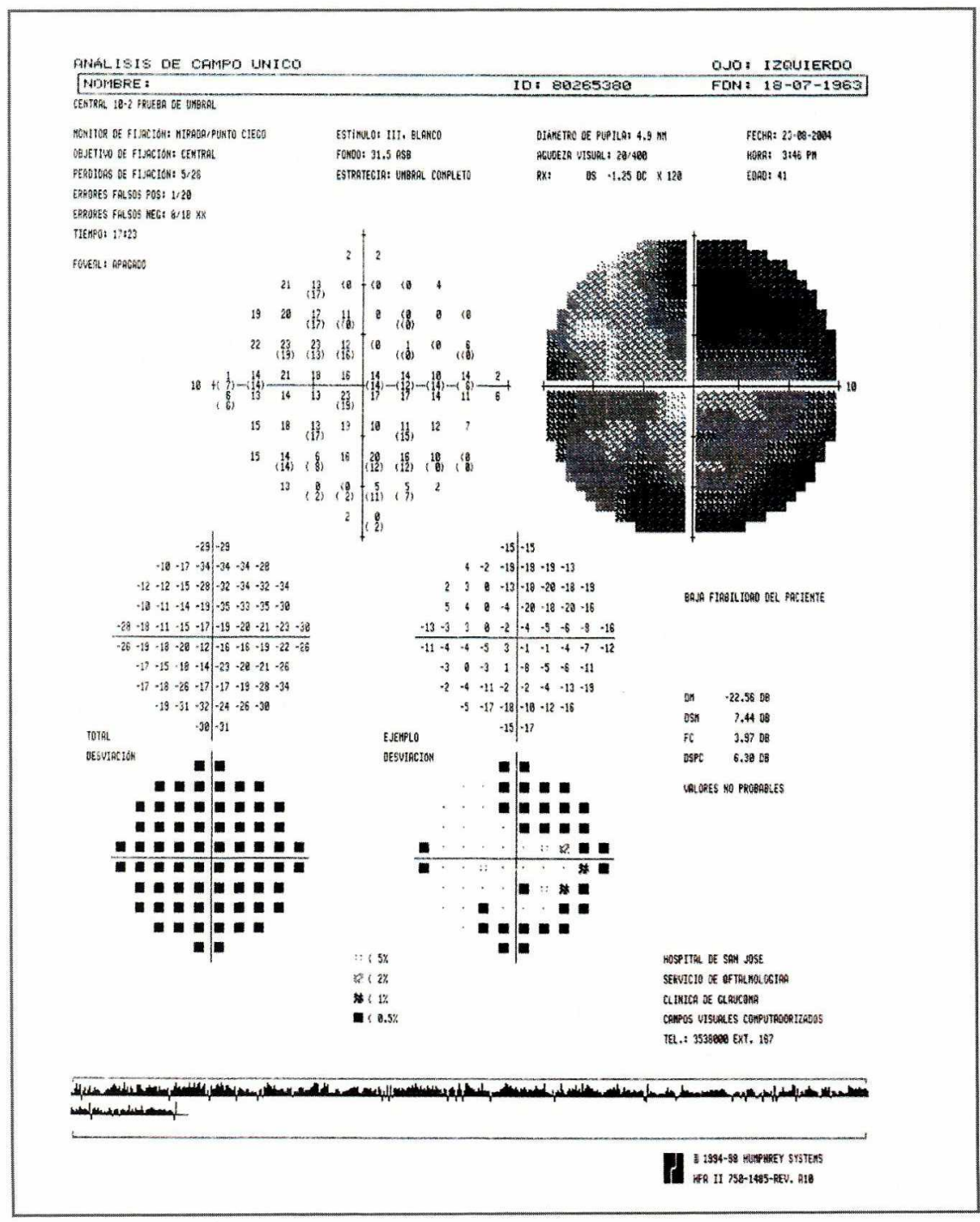

Campo visual 10-2 Ojo derecho 


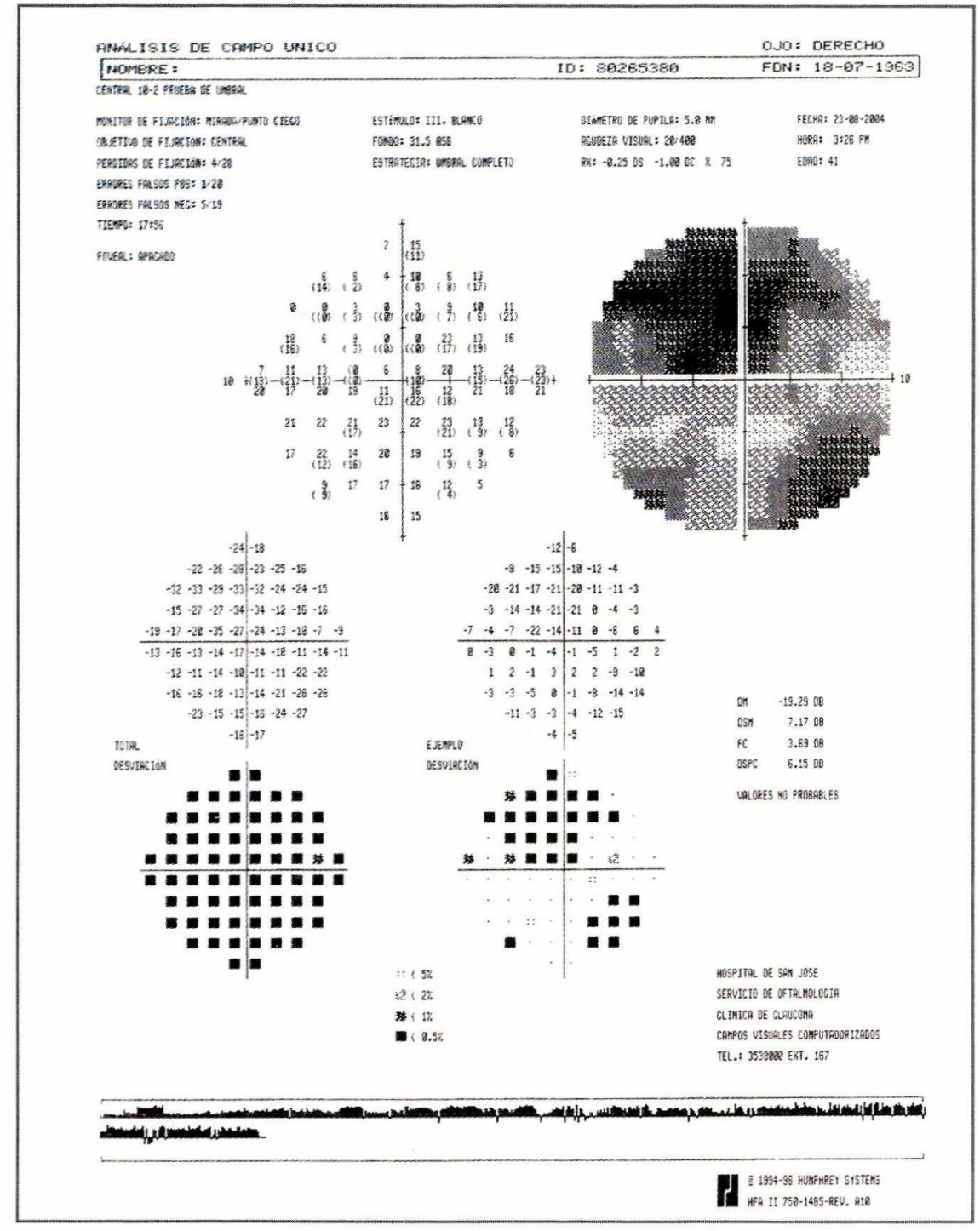

Campo visual 10-2 Ojo izquierdo

Figura 3. Campimetría 10-2 de ambos ojos donde se observa la gran disminución de la sensibilidad a nivel macular además de presentar escotomas en ambos ojos.

de genética realizar los estudios pertinentes para investigar las alteraciones cromosómicas de cada una de las patologías presentadas y sus posibles asociaciones.

\section{Revisión de la literatura}

\section{Distrofias corneanas}

Una distrofia es una alteración bilateral y simétrica en la mayoría de los casos, heredada, la cual no tiene relación con factores ambientales ni se asocia con hallazgos sistémicos. Las distrofias comienzan en los primeros años de vida, pero sus manifestaciones clínicas pueden presentarse después. La mayoría son lentamente progresivas. Se pueden clasificar por el patrón genético que presentan, su severidad, sus características histopatológicas o bioquímicas, pero la forma más sencilla y aceptada es la clasificación de acuerdo a la localización anatómica de éstas en la córnea.

\section{Distrofia polimorfa posterior de la córnea}

Descrita por primera vez por Koeppé en 1916, abarca una serie de anomalías en la córnea y el segmento anterior que van desde edema corneano congénito hasta 


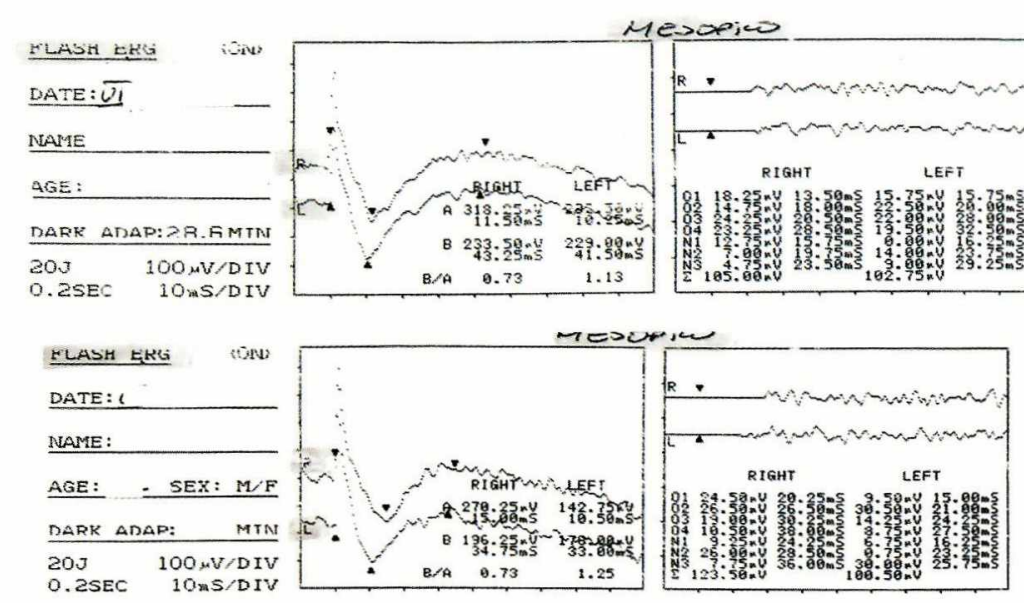

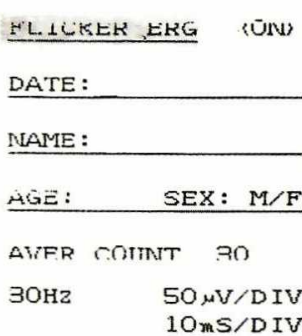

A

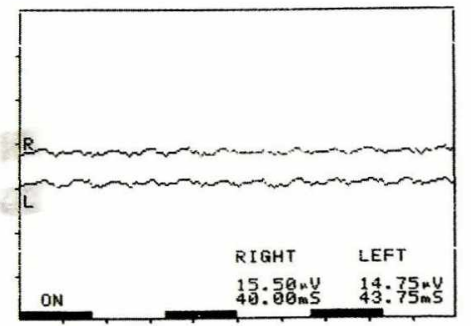

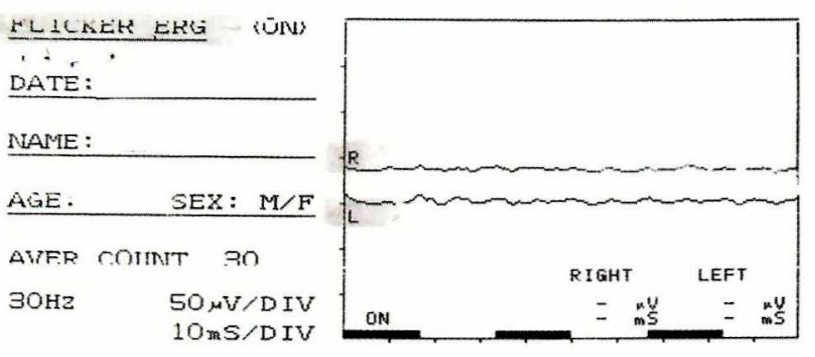

B
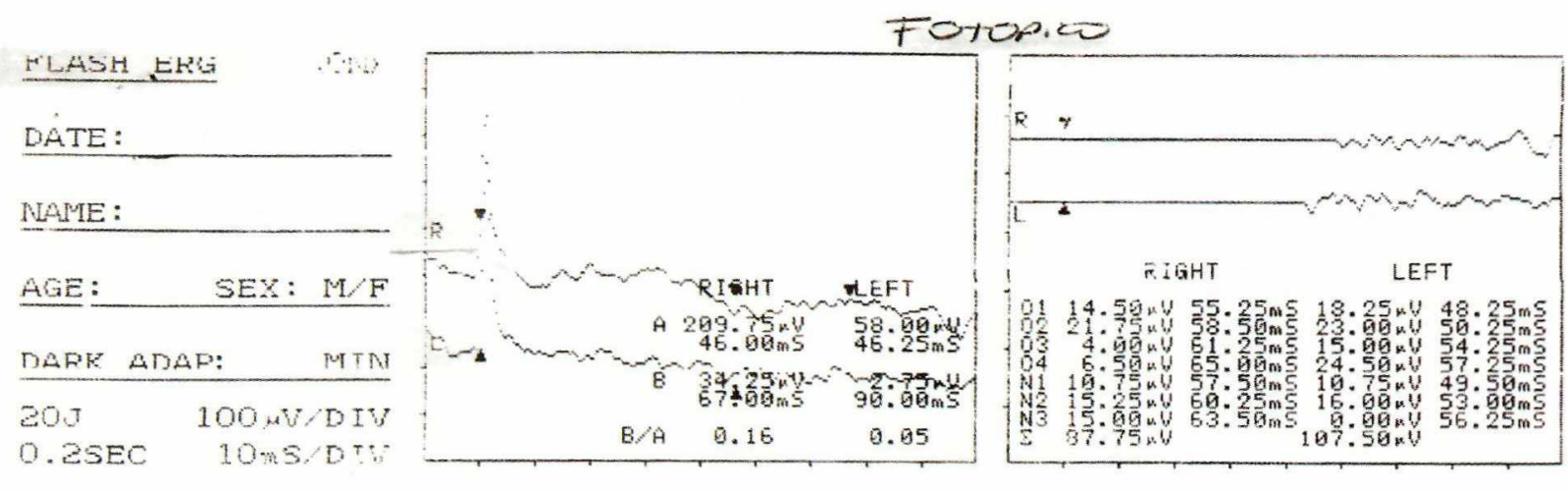

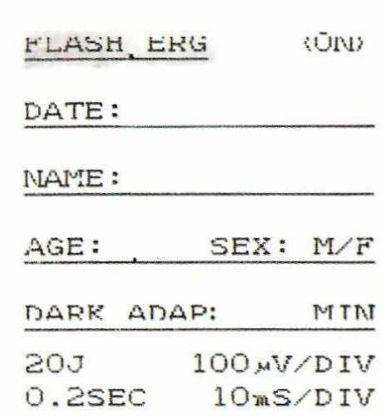
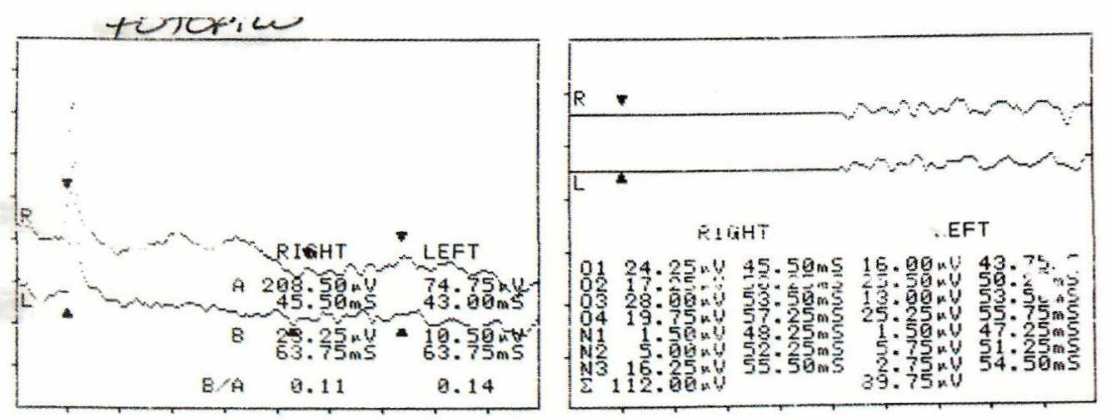

C

Figura 4. Electrorretinograma.

A. Porción mesópica. B. Porción Flicker C. Porción Fotópica. (Descripción en el texto.) 


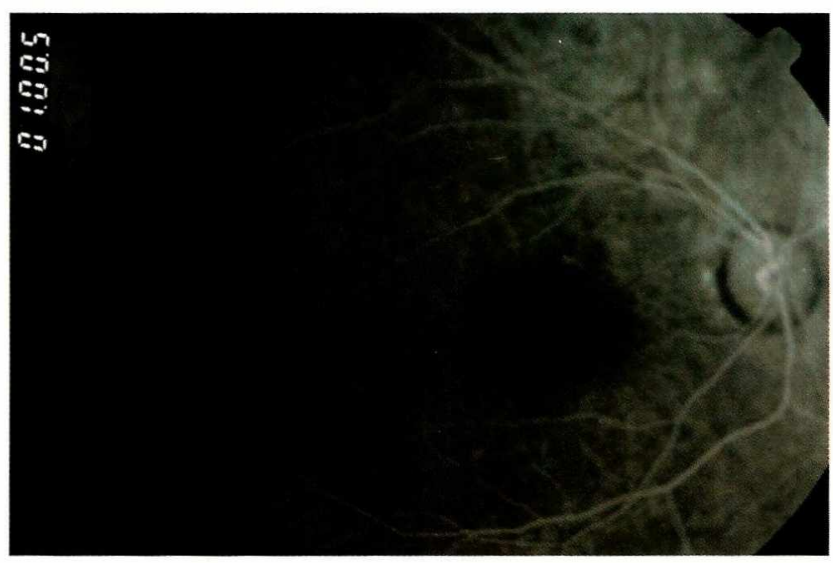

Fluoroangiografía ojo derecho

Figura 5. Angiografía fluoresceínica

edema corneano de aparición tardía en la edad media. Muchos de los casos son subclínicos y solo presentan pequeñas alteraciones en el examen biomicroscópico y en la microscopía especular. Es una patología autosómica dominante con alto grado de penetrancia (algunos casos muy extraños de patrón de herencia recesiva). Usualmente es bilateral y asimétrica, en algunos casos, el ojo contralateral puede presentar cambios mínimos catalogándola como unilateral. Presenta además alteraciones endoteliales y en la membrana de Descemet que cursan con grados variables de descompensación corneana. Puede asociarse con sinequias irido-corneales y glaucoma y tiene una tendencia a ser recurrente después de queratoplastia penetrante. Algunos de estos hallazgos se confunden con los encontrados en entidades como el síndrome irido-corneo-endotelial, la anomalía de Peter y el síndrome de Axenfeld Rieger, lo cual sugiere que la distrofia polimorfa posterior puede ser parte de un espectro de desórdenes que tienen en común alteraciones en la diferenciación final de las células de la cresta neural. Se ha informado asociación de la DPP con la degeneración posterior amiloide de la córnea, el queratocono y el síndrome de Alport, pero nunca ha sido reportada en la literatura asociada a distrofia retiniana de conos.

La distrofia polimorfa posterior puede ser congénita y presentarse con edema corneano al nacimiento. La edad de presentación en sujetos asintomáticos es desconocida y se cree que las alteraciones estén desde

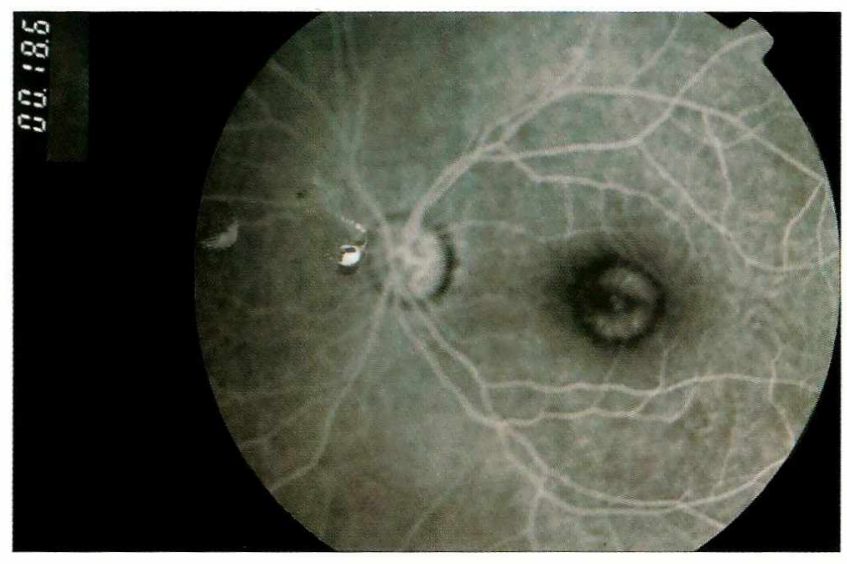

Fluoroangiografía ojo izquierdo

el nacimiento. Esta teoría está soportada por estudios ultraestructurales de la membrana de Descemet en la cual se observan áreas focales de atenuación en su capa anterior la cual se desarrolla antes del nacimiento, así como alteraciones en sus capas posteriores.

\section{Epidemiología}

La prevalencia en la población general es desconocida. Se ha observado que tiene un patrón hereditario autosómico dominante principalmente, sin embargo se han reportado casos autonómicos recesivos. La forma dominante se ha asociado a una alteración en el brazo largo del cromosoma 20 (20q11).

\section{Patogénesis}

A pesar de no ser clara aún, los resultados de estudios inmuno-histoquímicos han encontrado que las células endoteliales alteradas expresan antígenos propios del epitelio y por lo tanto se cree que la patología es causada por una metaplasia focal de células endoteliales en células aberrantes queratinizadas epiteliales.

\section{Histopatología}

La característica básica de la distrofia polimorfa posterior es la apariencia del endotelio que pareciera compuesto por células del epitelio, formando además varias capas. Estas células tienen microvellosidades, uniones por desmosomas y queratina. Tambien hay cambios 


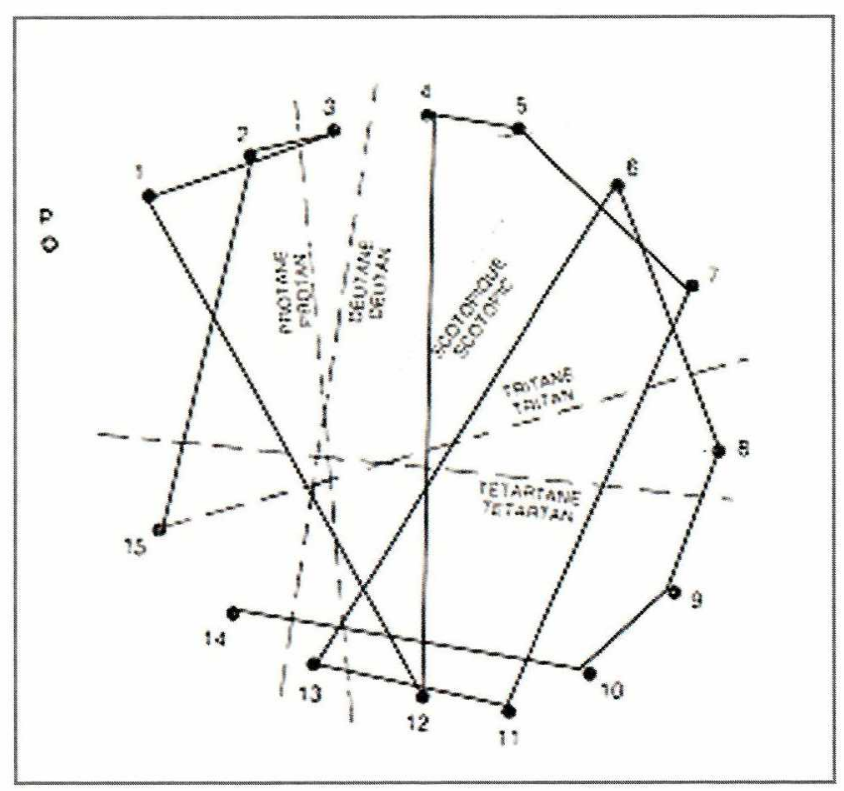

PRUEBA ESTÁNDAR OD

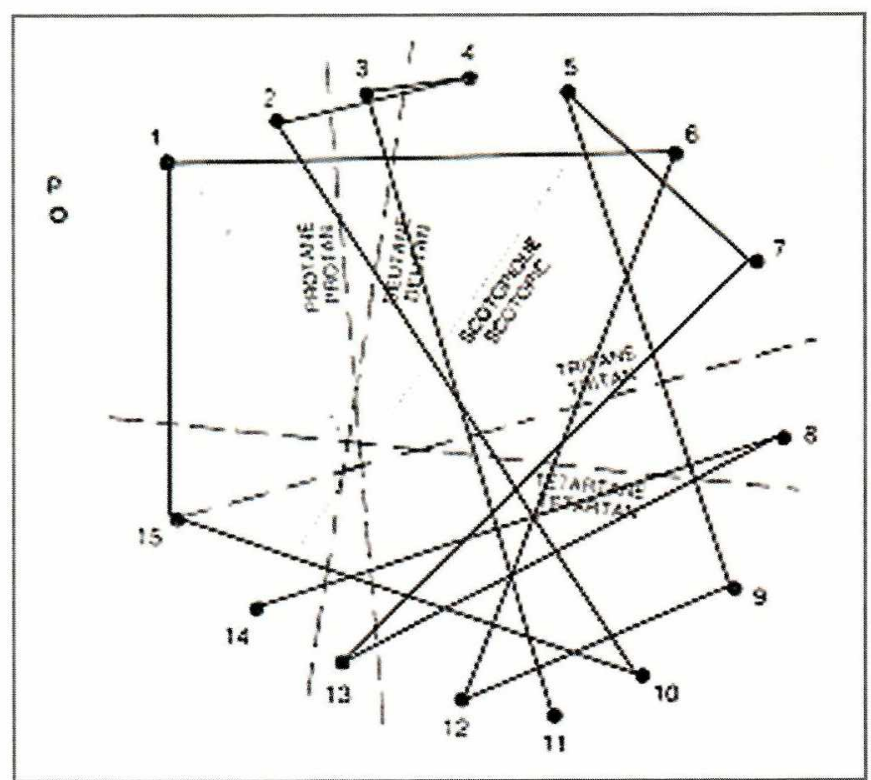

PRUEBA ESTÁNDAR OI

Figura 6. Farnsworth Munsell I5D donde se evidencia la discromatopsia presentada por el paciente.

tales como nódulos en el estroma profundo, crestas que forman un patron anular en el estroma posterior, opacidad difusa de las lamelas corneanas profundas y gutas. Por micorscopía especular se observan bandas y vesículas en por lo menos $50 \%$ de los casos, estos hallazgos ayudan a diferenciar la distrofia polimorfa posterior del síndrome irido-corneo-endotelial.

\section{Manifestaciones oculares}

Los hallazgos más comunes en el examen de lámpara de hendidura son vesículas aisladas bilaterales que parecen quistes rodeados de un halo gris a nivel de la membrana de Descemet. El tamaño de estas vesículas varía de 200 a 1000 micras. Pueden observarse algunas pocas o muchas, separadas o en grupos formando parches con patrón geográfico. Una presentación menos frecuente consiste en bandas o "huellas de caracol" de aproximadamente $1 \mathrm{~mm}$ de grosor y pueden tener una longitud de 2 a $10 \mathrm{~mm}$. En ambas formas, la vesicular y la linear, el estroma y el epitelio no están comprometidos y la visión es normal. La forma más rara de presentación es la difusa en la cual se compromete todo el endotelio. En esta última la visión por lo regular está afectada por edema corneano como resultado del compromiso difuso del endotelio y además puede asociarse con otras anormalidades del segmento anterior como presión intraocular elevada y sinequias iridocorneanas. Todos los pacientes con DPP tienen recuentos endoteliales menores a los controles normales para la edad.

\section{Diagnóstico}

Se hace con la observación clínica de las lesiones descritas en el examen biomicroscópico con la lámpara de hendidura. En los pacientes con edema corneano de origen desconocido, usualmente se hace el diagnóstico a través de estudio histopatológico del botón corneano después de una queratoplastia penetrante.

\section{Tratamiento}

La mayoría de pacientes no requieren tratamiento. En los casos de edema corneano leve, se puede manejar con la aplicación tópica de solución salina hipertónica o incluso con la utilización de un secador de cabello. Si presentan gran opacidad córneana que comprometa visión puede realizarse queratoplastia penetrante. En los casos que se asocian a glaucoma, adhesiones a iris o ambas, el pronóstico del tejido donante no es tan bueno como en los casos en las que no las hay. 


\section{Distrofia retiniana de conos}

Es una de las entidades pertenecientes al grupo de los trastornos del sistema de conos.

\section{Desórdenes del sistema de conos}

1. Pérdida de los conos

Congénitas hereditarias

Monocromatismo de bastones

Monocromatismo de conos

No congénitas hereditarias

Distrofia de conos

2. Anormalidades de los pigmentos de los conos

Discromatismo

Tricromatismo anómalo

En este trastorno no hay síntomas en el momento del nacimiento, puede ocurrir de manera esporádica o tener un patrón hereditario autosómico recesivo (cromosomas 2q11, 8q21, 1p13), dominante (cromosoma 17p) o ligado al cromosoma X. El diagnóstico es difícil debido a la gran variedad de cambios retinianos que pueden ocurren. La edad de inicio de los síntomas puede ser desde la primera década de la vida a la sexta, y los pacientes tienen visión normal antes de su presentación. Cuando comienza la sintomatología, típicamente los pacientes referirán perdida progresiva de la visión durante las horas del día, disminución de la agudeza visual y de la sensibilidad a los colores y fotofobia. Se deben realizar pruebas electrofisiológicas para confirmar el diagnóstico.

Hay varias formas conocidas de disfunción de conos, puede ser progresiva y no-progresiva. A continuación se enumeran síndromes de disfunción de conos:

1. Defectos congénitos de la visión de color sin ambliopía:

a. Transmisión ligada a X presenta deuteranopia y protanopia.

b. Transmisión autosómica dominante presenta tritanopia.
2. Ceguera al color completa sin ambliopía:
a. Monocromatismo
b. Patrón hereditario aún no establecido.

3. Ceguera al color incompleta congénita con agudeza visual subnormal:

a. Acromatopsia incompleta, probablemente ligada a X o autosómica recesiva.

4. Ceguera al color completa congénita con agudeza visual subnormal:

a. Acromatopsia completa con ambliopía

b. Monocromatismo de conos con transmisión autosómica recesiva.

5. Degeneraciones progresivas de los conos:
a. Patrón de transmisión desconocido.

6. Deficiencia generalizada de conos y bastones:

a. Los síntomas de la deficiencia de conos predominan.

7. Deficiencia generalizada de conos y bastones:

a. Los síntomas de la deficiencia de bastones predominan, como ejemplo la retinitis pigmentosa

Las alteraciones de los conos no congénitas que aparentemente sean heredadas son llamadas distrofia de conos, mientras que las de origen no claro se llaman degeneración de conos.

En algunos casos se ha reportado que no es posible diferenciar entre una degeneración de conos progresiva y una deficiencia generalizada de conos y bastones en la cual los síntomas relacionados con los conos predominan. Durante la vida puede haber una transición entre una y otra. La distrofia de conos pura se logró identificar en estudios en familias que presentaron un patrón hereditario autonómico dominante, aunque su presentación es fue muy variable. Sin embargo esta entidad presenta suficientes similitudes para definirla como una entidad única.

Aparte de la transmisión autosómica dominante, hay casos esporádicos de disfunción de conos adquirida así como de casos en los que hay patrón hereditario autonómico recesivo. La mayoría de estos tienen un compromiso difuso de los conos, pero en la mayoría éste es solo central. Esta alteración adquirida se puede demostrar en un electro- 
rretinograma al haber compromiso de la porción fotópica. La alteración de los conos se observaría con una disminución de la agudeza visual y una mala discriminación de colores.

El síntoma predominante es la perdida de agudeza visual, asociada casi siempre a fotofobia. A diferencia de otras maculopatías, en esta las alteraciones de la visión de color se presentan tempranamente. Los pacientes ven mejor en ambientes oscuros, prefieren usar anteojos oscuros y presentan los síntomas totalmente opuestos a un paciente con retinitis pigmentosa.

La perdida visual precede a los cambios que se observan en la fundoscopia. En el examen de fondo de ojo, se pueden observar cuatro tipos de lesiones diferentes:

Área de atrofia oval, bien definida, del epitelio pigmentario de la retina, rodeada de un área oscura homogénea a nivel central de la mácula que es llamada en "ojo de buey". Es similar a la observada en los pacientes manejados con cloroquina.

Pigmento en puntos y difuso, en grupos en el polo posterior. Es una forma menos frecuente de presentación. Suele verse en familias con patrón de herencia autonómico recesivo.

- Vasos coroideos grandes con atrofia de la coriocapilaris.

- Cambios en la fundoscopia como los encontrados en el fondo flavimáculatus y en la enfermedad de Stargardt.

En todas estas formas variables de presentación puede haber atrofia de nervio óptico, atenuación vascular y espículas de hueso similares a las vistas en la retinitis pigmentosa.

Los cambios en el fondo de ojo determinarán la presentación de una angiografía fluoresceínica. Si el patrón se presenta en "ojo de buey", se observará un área oval de hiperfluorescencia rodeando un centro hipofluorescente. En la atrofia difusa de conos se verá una hiperfluorescencia difusa en el polo posterior con bordes bien definidos con áreas de no fluorescencia. En el grupo en que hay atrofia de la coriocapilaris se observarán cambios como los encontrados en una atrofia coroidea central. En estadíos tardíos en los que hay compromiso de bastones, el patrón angiográfico recordará aquel de retinitis pigmentosa.

Hay diversas pruebas de función de la retina en la cuales cada una presentará cambios patológicos.

Agudeza visual: la agudeza visual típicamente sufre un deterioro progresivo.

Campos visuales: la campimetría puede ser normal en la periferia, pero mostrará un escotoma central. La periferia se puede ver comprometida en casos severos y muy avanzados, en los cuales habrá constricción del campo visual.

Visión de color: los pacientes presentan múltiples errores en las pruebas para color como el Ishihara y el Farnsworth 100 o 15D.

Electrorretinograma: la porción flash sencillo - fotópica generalmente es muy baja o imposible de registrar, la fotópica flicker está ausente o da pequeñas respuestas. La porción escotópica es normal cuando el compromiso está limitado a los conos.

Electro-oculograma: suele ser normal.

El diagnóstico diferencial se debe hacer con otras distrofias maculares, como la enfermedad de Stargardt, distrofia macular atrófica progresiva, distrofia viteliforme, distrofia pseudoinflamatoria y degeneración macular miopica, entre otras. El diagnóstico final se logra interpretando los datos obtenidos en la historia clínica, el examen oftalmológico completo y el resultado de pruebas auxiliares que valoren la función de la retina. 


\section{Lecturas recomendadas}

- American Academy of Ophthalmology 2003 - 2004. Basic and clinical science course. Section 8 2003-2004: external disease and cornea. San Francisco: American Academy of Ophthalmology; 2004.

- Grupcheva CN, Chew GS, Edwards M, Craig JP, McGhee. Imaging posterior polymorphous corneal dystrophy by in vivo confocal microscopy. Clin Experiment Ophthalmol. 2001 Aug; 29(4):256-9.

- Kaufman HE, Barron BA, McDonald, MB, editores. The Cornea. 2nd ed. [New York]: Butterworth Heinmann, 1997.

- Klintworth GK. The molecular genetics of the corneal dystrophies - current status. Front Biosci. 2003 May 1; 8: 687-713.

- Krachmer JH, Mannis MJ, Holland EJ, Palay DA, editors. Córnea text \& color atlas [CD-ROM]. St Louis : Mosby; 1998.
- PubMed [base de datos en Internet]. National Library of Medicine. C2007. . [citado 17 Mar 2007]. Disponible en: www.pubmed.com

- Regillo CD, editor. Basic and clinical science course. Section 12 2003-2004: retina and vitreous. San Francisco: American Academy of Ophthalmology; 2006.

- Ryan SJ, Hinton DR, Schachat AP. Retina [CD-ROM]. 3rd ed. St Louis : Mosby; 1995.

- $\quad$ Smolin G, Thoft R. The cornea, 3rd ed. [s.1.]: Little, Brown and Company, 1994.

- Tasman W, Jaeger EA. editores. Duane's clinical ophthalmology [CD-ROM]. Philadelphia: Lippincott, Williams \& Wilkins, 2003.

- Voo I, Small KW.. Update on the genetics of macular dystrophies.Retina. 2004 Aug; 24(4):591-601.

- Yanoff M, Duker JS. Ophthalmology [monografía en Internet]. 2nd ed. [Washington]: Elsevier; 2004. [citado 17 Mar 2007]. Disponible en: http://www.ophtext.com/

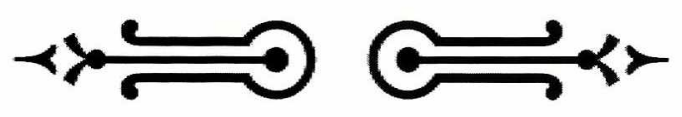

